\section{Commentary: The body's innate immune response to transcatheter aortic valve implantation manifesting in the bloodstream- complement activation gone wrong!}

\author{
Victor A. Ferraris, MD, PhD
}

The human body has all kinds of responses at its disposal to recognize and to respond to cellular and infectious dangers. Some of those responses are directed at abnormal substances in the bloodstream. These responses are associated with innate immunity and consist of at least 5 responses, including fever, inflammation, phagocytosis, interferon production, and complement activation.

The complement system detects and removes foreign, potentially harmful substances. This system is a complex conglomerate of 30 proteins that act in concert to help eliminate infectious microorganisms or potentially harmful foreign substances from the bloodstream. Specifically, the complement system causes the lysis of foreign and infected cells, the phagocytosis of foreign particles and cellular debris, and the inflammation of surrounding tissue. Since 1901, Nobel Prizes in Physiology and Medicine have been awarded to 11 different groups of investigators who contributed to our understanding of how foreign substances and organisms are recognized and removed to prevent damage to important bodily systems. ${ }^{1}$

The complement system is not always associated with benefit. Veraar and colleagues ${ }^{2}$ describe a case of the

\footnotetext{
From the Division of Cardiothoracic Surgery, University of Kentucky, and Lexington VA Medical Center, Lexington, Ky.

Disclosures: The author reported no conflicts of interest.

The Journal policy requires editors and reviewers to disclose conflicts of interest and to decline handling or reviewing manuscripts for which they may have a conflict of interest. The editors and reviewers of this article have no conflicts of interest.

Received for publication March 9, 2021; accepted for publication March 10, 2021; available ahead of print April 5, 2021.

Address for reprints: Victor A. Ferraris, MD, PhD, Division of Cardiothoracic Surgery, University of Kentucky, A301 Kentucky Clinic, 740 S Limestone, Lexington, KY 40536-0284 (E-mail: Ferraris@uky.edu).

JTCVS Open 2021;6:99-100

2666-2736

Copyright (c) 2021 The Author(s). Published by Elsevier Inc. on behalf of The American Association for Thoracic Surgery. This is an open access article under the CC BY-NC-ND license (http://creativecommons.org/licenses/by-nc-nd/4.0/)

https://doi.org/10.1016/j.xjon.2021.03.004
}

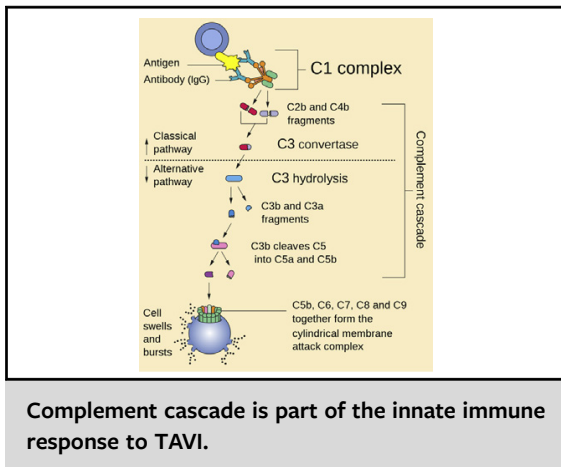

CENTRAL MESSAGE

The body's response to TAVI

may be compromised by its

innate immune response.

complement system gone wrong. ${ }^{2}$ These authors studied 27 patients who underwent transcatheter aortic valve implantation (TAVI) and measured components of the immune system 3 months after TAVI. They used serum enzymelinked immunosorbent assay to measure various components of the complement system and other allergens. They found substantially increased concentrations of the following in the bloodstream of patients undergoing TAVI 90 days after valve implantation.

- Alpha-gal-specific immunoglobulin G3 Alpha-gal syndrome (ie, red meat allergy) is a recently identified allergy to red meat and other mammalian products. In the United States, the condition most often begins from a Lone Star tick bite. The bite transmits a sugar molecule called alpha-gal into the person's body. In some people, this triggers an immune system reaction that later produces mild to severe allergic reactions to red meat.

- Complement factor C3a C3a represents an effector of the complement system with a wide range of functions, including T-cell activation, angiogenesis, chemotaxis, and macrophage activation.

- Citrullinated histone H3 Citrullinated histone H3 is released into the blood from neutrophil extracellular traps in response to severe infection, and citrullinated histone $\mathrm{H} 3$ may be a potential biomarker for sepsis. Neutrophil extracellular traps are networks of extracellular fibers, primarily composed of DNA from lysed neutrophils that bind pathogens.

- Soluble suppression of tumorigenicity-2 Soluble suppression of tumorigenicity-2 is upregulated in response to acute myocardial infarction and may represent a 
clinically relevant biomarker for predictive information in the setting of several cardiovascular diseases, and especially in patients with heart failure.

At this stage of the investigations, it is not possible for the authors to clearly define the long-term aggregate actions of these 4 substances and the observations have to be considered very preliminary. It is entirely possible that the surface of a transcatheter aortic valve replacement (TAVR) valve will become passivated as time goes on after implantation. Similar assessments of TAVR valves, like those done by the authors, have not been done over much longer and more extended postoperative periods.

Perhaps among the most unique and actionable findings of the study is the identification of at least 4 possible targets for modification of TAVR valves that may improve longer-term valve survival with good function. The authors have not identified future plans for study of passivation of TAVR valves, or for traditional tissue prosthetic valves, for that matter. However, a possible next step may be development of methods to create genetically modified TAVR valves that are free from the 4 immune inflammatory moderators found in the authors' preliminary experiments. It is intriguing to speculate that molecular modifications of valve tissue may hold promise for the creation of a durable and possibly novel type of bioprostheses with much longer durability and survival in humans.

\section{References}

1. Nobel prizes and the immune system. Available at: https://www.nobelprize.org/ prizes/uncategorized/nobel-prizes-and-the-immune-system. Accessed March 16, 2021.

2. Veraar C, Koschutnik M, Nitsche C, Laggner M, Polak D, Bohle B, et al. Inflammatory immune response in recipients of transcatheter aortic valves. J Thorac Cardiovasc Surg Open. 2021;6:85-96. 